\title{
Probing mechanical properties of living cells by atomic force microscopy with blunted pyramidal cantilever tips
}

\author{
Félix Rico, Pere Roca-Cusachs, Núria Gavara, Ramon Farré, Mar Rotger, and Daniel Navajas* \\ Unitat de Biofísica i Bioenginyeria, Facultat de Medicina, Universitat de Barcelona-IDIBAPS, \\ Casanova 143, 08036 Barcelona, Spain \\ (Received 4 April 2005; published 29 August 2005)
}

\begin{abstract}
Atomic force microscopy (AFM) allows the acquisition of high-resolution images and the measurement of mechanical properties of living cells under physiological conditions. AFM cantilevers with blunted pyramidal tips are commonly used to obtain images of living cells. Measurement of mechanical properties with these tips requires a contact model that takes into account their blunted geometry. The aim of this work was to develop a contact model of a blunted pyramidal tip and to assess the suitability of pyramidal tips for probing mechanical properties of soft gels and living cells. We developed a contact model of a blunted pyramidal tip indenting an elastic half-space. We measured Young's modulus $(E)$ and the complex shear modulus $\left(G^{*}=G^{\prime}+\mathrm{i} G^{\prime \prime}\right)$ of agarose gels and A549 alveolar epithelial cells with pyramidal tips and compared them with those obtained with spherical tips. The gels exhibited an elastic behavior with almost coincident loading and unloading force curves and negligible values of $G^{\prime \prime} . E$ fell sharply with indentation up to $\sim 300 \mathrm{~nm}$, showing a linear regime for deeper indentations. A similar indentation dependence of $E$ with twofold lower values at the linear regime was obtained with the spherical tip fitted with Hertz's model. The dependence of $E$ on indentation in cells paralleled that found in gels. Cells exhibited viscoelastic behavior with $G^{\prime \prime} / G^{\prime} \sim 1 / 4$. Pyramidal tips commonly used for AFM imaging are suitable for probing mechanical properties of soft gels and living cells.
\end{abstract}

DOI: 10.1103/PhysRevE.72.021914

PACS number(s): $87.64 . \mathrm{Dz}, 87.19 . \mathrm{Rr}, 87.80 . \mathrm{Tq}$

\section{INTRODUCTION}

The mechanical properties of the cell play a key role in determining cell shape and changes during essential functions such as contraction, crawling, and migration. Microscopy and nanomanipulation techniques have led to a dramatic increase in our knowledge of the morphology and mechanical properties of cells. One of the most powerful tools is atomic force microscopy (AFM), which allows us to obtain topographical images and to probe mechanical properties of living cells under physiological conditions [1]. Moreover, AFM can be used to monitor dynamic changes in the shape and mechanics of the cell during pharmacological treatments.

AFM uses a flexible cantilever with a sharp tip at its end to probe the sample surface. Images are obtained by scanning the surface of the sample with the cantilever tip. The sharpness of the tip, apart from other characteristics such as sample stiffness, determines image resolution [2]. Soft silicone nitride AFM cantilevers with pyramidal tips are commonly used for obtaining high-resolution images of living cells $[3,4]$. Pyramidal tips are blunted or rounded at the apex. Despite their bluntness, they are sharp enough to resolve subcellular structures of living cells, such as the cytoskeleton, which plays the main role in cellular mechanics [5-10].

The cell mechanics are probed with AFM by indenting the surface of the cell with the tip of the cantilever and measuring the force-indentation $(F-\delta)$ relationship. The mechanical parameters of the sample are usually estimated by fitting

\footnotetext{
*Corresponding author. Electronic address: dnavajas@ub.edu, http://www.ub.edu/biofisica
}

$F$ - $\delta$ data with a suitable contact model, taking into account the geometry of the probe. Hertz's theory [11] is the most common approach to analyzing contact mechanics. This approach is based on infinitesimal strains and assumes linear elastic, isotropic, axisymmetric, perfectly smooth, and semiinfinite bodies in contact with each other over a small region of their surface [11]. In particular, Hertz's theory leads to analytical solutions for spherical and conical tips indenting an elastic half-space [12]. Assuming a spherical geometry of the tip apex, the spherical Hertz model has been applied to pyramidal tips to determine the mechanical properties of biopolymer gels and living cells [13-15]. This model fails for indentations larger than the apparent radius of curvature of the tip $(10-50 \mathrm{~nm})$. For deep indentations, pyramidal tips have been modeled as cones $[13,16]$. A blunted cone model has been developed for a wider range of indentations $[17,18]$. However, the actual geometry of pyramidal tips is not a blunted cone but a blunted pyramid. A contact model for an ideal pyramid has been numerically developed by Bilodeau [19]. This pyramidal model has recently been used to probe the mechanical properties of living cells at deep indentations [20]. To the best of our knowledge, a more detailed pyramidal contact model, taking into account the blunt geometry of conventional AFM pyramidal tips, has not been developed.

The use of a cantilever with a microsphere attached at its end has recently been proposed to indent a sample with a simple contact geometry and to minimize strain $[21,22]$. Cantilevers with microspheres ranging $\sim 1$ to $\sim 10 \mu \mathrm{m}$ in diameter have been used to probe biopolymer gels and living cells [21-24]. Sample mechanical properties were estimated by fitting indentation data with the Hertz spherical model. Although a microsphere provides a simple contact geometry for probing mechanics, the use of this tip yields low- 
resolution images that may not reflect the actual topography of some samples [25]. Replacing the cantilever between imaging and mechanical measurements is difficult and time consuming. This may be suitable for large, stable, and uniform samples but not for living cells, since they have a complex shape and exhibit heterogeneous and time-varying mechanical properties. Moreover, the mechanics and shape of the cell change during spontaneous contraction and locomotion and in response to pharmacological stimuli. The use of a single cantilever for imaging and indentation enables us to precisely correlate the shape and mechanics of living cells. Pyramidal tips commonly used for AFM imaging could be suitable for probing cell mechanics. Moreover, measurements at low indentations could be improved by developing a blunted pyramidal model that takes into account the blunted geometry of the tip.

The aim of this work was to develop a contact model of a blunted pyramidal tip and to assess the suitability of AFM pyramidal tips for probing mechanical properties of soft biopolymer gels and living cells. We first developed a contact model of a blunted regular pyramid indenting an elastic half-space. We probed soft samples of agarose gels and living human alveolar epithelial cells with pyramidal and spherical tips using the blunted pyramidal model and the spherical Hertz model, respectively. The Young's modulus $(E)$ and the complex shear modulus $\left[G^{*}(\omega)\right]$ estimated with the pyramidal and spherical tips were compared.

\section{THEORY}

\section{Contact model of a blunted pyramid indenting an elastic half-space}

The force-indentation relationship, $F(\delta)$, for a blunted $n$-sided rigid regular pyramid of semi-included angle $\theta$, tip defect $h$, and spherical cap radius $R_{c}$ (Fig. 1) was derived using Betti's reciprocal theorem [26]. Accordingly, the total force $F$ applied by a tip with an arbitrary shape and projected area $A$ was computed from the pressure distribution of a flat indenter $\left[p^{*}(r, \varphi)\right]$ of plan form $A$

$$
F=\iint_{A} p^{*}(r, \varphi) \frac{f(r, \varphi)}{\delta^{*}} r d r d \varphi
$$

where $\delta^{*}$ is the indentation depth for the flat indenter, and $f(r, \varphi)$ is the interpenetration function for the tip. The analytical form of $p^{*}(r, \varphi)$ is known only for elliptical contact areas. Thus, only in this case can a closed-form solution of Eq. (1) be obtained. Nevertheless, $p^{*}(r, \varphi)$ and therefore $F$ can be approximated in the Rayleigh-Ritz sense, i.e., by using the best elliptical approximation to the actual contact area [27].

For a regular $n$-sided pyramid, the cross section at any indentation depth is a regular $n$-sided polygon. Therefore, its best elliptical approximation is a circle of radius $a$ centered on the axis of the pyramid, and the pressure distribution of the corresponding flat indenter is that of a cylindrical punch of radius $a$
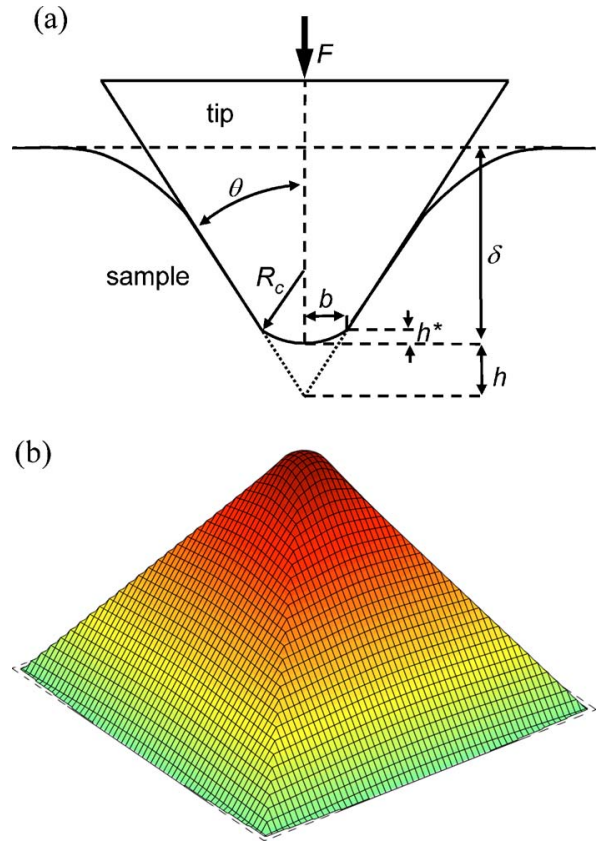

FIG. 1. (Color online) Model of the blunted pyramidal AFM tip. (a) Section of a blunted pyramidal tip with semi-included angle $\theta$, tip defect $h$, and spherical cap radius $R_{c}$ indenting an elastic halfspace. $F$ is force, $\delta$ is indentation, $b$ is the radial distance corresponding to the transition from spherical cap to pyramidal faces, and $h^{*}=b^{2} / 2 R_{c}$. (b) Three-dimensional plot of the end $(1 \mu \mathrm{m})$ of the blunted four-sided pyramidal tip model with the spherical cap merging tangential with the pyramid faces $\left(\theta=35^{\circ}, R_{c}=100 \mathrm{~nm}\right)$. Dashed line is the contour corresponding to flat faces (ideal pyramid).

$$
p^{*}(r, \varphi)=\frac{E}{\pi\left(1-\nu^{2}\right)} \frac{\delta^{*}}{\left(a^{2}-r^{2}\right)^{1 / 2}} .
$$

Substituting this expression into Eq. (1), we obtain

$$
F=\frac{E}{\pi\left(1-\nu^{2}\right)} \iint_{A} \frac{f(r, \varphi)}{\left(a^{2}-r^{2}\right)^{1 / 2}} r d r d \varphi
$$

where $E$ is Young's modulus, and $\nu$ is Poisson's ratio.

The blunted pyramid was modeled as a spherical cap that transforms smoothly into an $n$-sided pyramid (Fig. 1) according to the following interpenetration function:

$$
\begin{gathered}
f(r)=\delta-\frac{r^{2}}{2 R_{c}} \quad 0 \leqslant a<b, \\
f(r, \varphi)=\delta-\frac{r-b}{\tan \theta} \cos \left(\varphi-\frac{2 k \pi}{n}\right)-h^{*}, \\
a \geqslant b, \frac{(2 k-1) \pi}{n}<\varphi<\frac{(2 k+1) \pi}{n}, \quad k=0,1, \ldots, n-1,
\end{gathered}
$$

where $b$ is the radial distance corresponding to the transition from spherical cap to pyramidal faces and $h^{*}=b^{2} /\left(2 R_{c}\right)$. For the particular case in which the sphere merges tangential with the pyramid faces, $b=R_{c} \cos \theta$, and the geometrical parameters of the tip are reduced to $R_{c}$ (or $b$ ) and $\theta$. 
For $a<b, f(r)$ is the interpenetration function of a spherical punch of radius $R_{c}$, and thus $F(\delta)$ is the spherical Hertz model [11]

$$
F=\frac{4 E}{3\left(1-\nu^{2}\right)} R_{c}^{1 / 2} \delta^{3 / 2}
$$

For $a>b$,

$$
\begin{aligned}
F= & n \frac{E}{\pi\left(1-\nu^{2}\right)}\left[\int_{0}^{b} \int_{-\pi / n}^{\pi / n}\left(\delta-\frac{r^{2}}{2 R_{c}}\right) \frac{r d r d \varphi}{\left(a^{2}-r^{2}\right)^{1 / 2}}\right. \\
& \left.+\int_{b}^{a} \int_{-\pi / n}^{\pi / n}\left(\delta-\frac{r-b}{\tan \theta} \cos \varphi-h^{*}\right) \frac{r d r d \varphi}{\left(a^{2}-r^{2}\right)^{1 / 2}}\right] \\
= & \frac{2 E}{1-\nu^{2}}\left\{\delta a-\frac{n}{\pi} \sin \left(\frac{\pi}{n}\right) \frac{a^{2}}{2 \tan \theta}\left(\frac{\pi}{2}-\arcsin \frac{b}{a}\right)-\frac{a^{3}}{3 R_{c}}\right. \\
& \left.+\left(a^{2}-b^{2}\right)^{1 / 2}\left[\frac{n}{\pi} \sin \left(\frac{\pi}{n}\right) \frac{b}{2 \tan \theta}+\frac{a^{2}-b^{2}}{3 R_{c}}\right]\right\} .
\end{aligned}
$$

Owing to the symmetry of the $n$ lateral edges, the contribution of each integration range $[(2 k-1) \pi / n,(2 k+1) \pi / n]$ is equal, and the integration over $\varphi$ is $n$ times the integration over the first range. The effective contact radius $a$ is obtained by imposing $\partial F / \partial a=0$

$$
\delta-\frac{a}{\tan \theta} \frac{n}{\pi} \sin \left(\frac{\pi}{n}\right)\left(\frac{\pi}{2}-\arcsin \frac{b}{a}\right)+\frac{a}{R_{c}}\left[\left(a^{2}-b^{2}\right)^{1 / 2}-a\right]=0 .
$$

Equations (6) and (7) determine the relationship $F(\delta)$ for a blunted $n$-sided regular pyramid. The indentation is related to the effective radius of contact $a$ by Eq. (7).

For the common regular four-sided pyramid of AFM cantilever tips, the preceding equations simplify to

$$
\begin{gathered}
F=\frac{2 E}{1-\nu^{2}}\left[\delta a-\frac{2^{1 / 2}}{\pi} \frac{a^{2}}{\tan \theta}\left(\frac{\pi}{2}-\arcsin \frac{b}{a}\right)-\frac{a^{3}}{3 R_{c}}\right. \\
\left.+\left(a^{2}-b^{2}\right)^{1 / 2}\left(\frac{2^{1 / 2}}{\pi} \frac{b}{\tan \theta}+\frac{a^{2}-b^{2}}{3 R_{c}}\right)\right], \\
\delta-\frac{a}{\tan \theta} \frac{2^{3 / 2}}{\pi}\left(\frac{\pi}{2}-\arcsin \frac{b}{a}\right)+\frac{a}{R_{c}}\left[\left(a^{2}-b^{2}\right)^{1 / 2}-a\right]=0 .
\end{gathered}
$$

In the limit $b \rightarrow 0$, Eqs. (8) and (9) lead to $F(\delta)$ for an ideal regular four-sided pyramid

$$
F=\frac{1}{2^{1 / 2}} \frac{E \tan \theta}{\left(1-\nu^{2}\right)} \delta^{2},
$$

with an effective radius of contact $a=\delta \tan \theta / 2^{1 / 2}$.

\section{MATERIALS AND METHODS}

\section{A. Atomic force microscopy}

Measurements were carried out with an AFM (Bioscope, Digital Instruments, Santa Barbara, CA, USA), mounted on an inverted optical microscope (IX70, Olympus Optical Co., Tokyo, Japan). V-shaped Au-coated silicon nitride cantilevers with nominal spring constant $k=10 \mathrm{mN} / \mathrm{m}$ with a regular four-sided pyramidal tip of nominal semi-included angle $\theta$ $=35^{\circ}$ (Microlevers, Thermomicroscopes, Sunnyvale, CA, USA), and similar cantilevers with a spherical polystyrene bead with nominal diameter of $5 \mu \mathrm{m}$ glued at their end (NovaScan Technologies, Ames, Iowa, USA) were used as AFM probes. One pair of pyramidal and spherical cantilevers was used for probing agarose gels, and another pair for probing cells. The cantilevers were cleaned before each set of measurements, using piranha solution $\left(70 \% \mathrm{H}_{2} \mathrm{SO}_{4}, 30 \%\right.$ $\mathrm{H}_{2} \mathrm{O}_{2}$ ) for the pyramidal tips and ethanol for the spherical ones. Scanning electron microscopy (SEM, Zeiss DSM 940-A, Zeiss, Göttingen, Germany) images of the cantilevers were obtained after measurements to determine the actual tip geometries.

\section{B. Calibration of cantilever spring constants}

The spring constants of the cantilevers were determined by the thermal fluctuations method [28]. Briefly, the cantilever was modeled as a simple harmonic oscillator. According to the equipartition theorem, each averaged quadratic term of the Hamiltonian of the system is given by $k_{B} T / 2$, where $k_{B}$ is the Boltzmann constant, and $T$ is the absolute temperature. Three seconds of cantilever thermal fluctuations were recorded in air away from any surface at room temperature. Data were filtered with an antialiasing filter (Butterworth, $106 \mathrm{kHz}, 8$ poles) and sampled at $500 \mathrm{kHz}$ by a computer interface board (PCI-MIO-16E-4, National Instruments, Austin, TX, USA). The power spectrum of fluctuation recordings was computed by fast Fourier transform (FFT). The first resonant peak was fitted with a Lorentzian curve plus an offset to reject contributions other than the first oscillation mode due to white noise. The mean square of fluctuations was computed as the area under the curve $(P)$. Invoking the Parseval theorem, the spring constant was computed as $k$ $=c k_{B} T / P$, where $c$ is a correction factor $c=0.76$ for the first vibration mode of a V-shaped cantilever [29].

\section{Agarose gel preparation}

Purified agarose (Type I-A: Low EEO, A-0169, Sigma, St. Louis, MO, USA) of $0.3 \% \mathrm{w} / \mathrm{v}$ in Millipore RX water was boiled for $\sim 20 \mathrm{~min}$, stirring continuously. The agarose solution was poured into a 35-mm-diam Petri dish to obtain a gel thickness of $\sim 500 \mu \mathrm{m}$. A small region of the Petri dish was left bare to allow calibration of the AFM photodiode. After gelation of the solution occurs $(\sim 5 \mathrm{~min})$, the sample was covered with $2 \mathrm{ml}$ of Millipore RX water and stored at $4{ }^{\circ} \mathrm{C}$.

\section{Cell culture}

Cell measurements were carried out in living human alveolar epithelial cells, line A549 (CCL-185, ATCC, Manassas, VA, USA). The culture medium consisted of HEPES (Sigma Chemical, St. Louis, Missouri) buffered RPMI 1640 with $10 \%$ inactivated fetal calf serum (Biological Industries, Kibbutz Beit Haemek, Israel), 1 mM L-glutamine, 100 U/ml 
penicillin, $100 \mathrm{mg} / \mathrm{ml}$ streptomycin (GIBCO, Gaithersburg, $\mathrm{MD}$, USA), and $2 \mathrm{mg} / \mathrm{ml}$ amphotericin B (Bristol-Myers Squibb Co., New Brunswick, NJ, USA). The cells were incubated at $37{ }^{\circ} \mathrm{C}$ and $5 \% \mathrm{CO}_{2}$. Two days before the experiments, the cells were trypsinized and plated on 10-mm-diam glass coverslips. The culture medium used during experiments was serum free.

\section{E. Measurements}

A Petri dish with agarose gel was placed on the temperature-controlled stage (Lake Shore Cryotronics, Westerville, $\mathrm{OH}, \mathrm{USA}$ ) of the AFM at $26^{\circ} \mathrm{C}$ and stabilized for $\sim 30$ min with the cantilever immersed in liquid. The AFM photodiode was calibrated by recording three force curves in the bare region of the Petri dish using a deflection range $\sim 1 \mu \mathrm{m}(F \approx 10 \mathrm{nN})$. Ten deflection-distance $(d-z)$ curves (triangular oscillations at $0.3 \mathrm{~Hz}$ of $3 \mu \mathrm{m}$ peak-to-peak amplitude) were recorded (2048 points/cycle) in three different regions of each gel. The cantilever was approached until reaching a maximum indentation $\sim 2 \mu \mathrm{m}$ and $\sim 1 \mu \mathrm{m}$ for the pyramidal and spherical tips, respectively. After the last $d-z$ measurement, low-amplitude triangular oscillations (100 nm peak-to-peak, $0.3 \mathrm{~Hz}$ ) were applied at an operating indentation of $\sim 500 \mathrm{~nm}$, and five cycles were recorded at 128 points/cycle. Measurements were carried out on seven agarose gel samples. Each sample was probed with both pyramidal and spherical tips in random order.

A similar protocol of measurements was taken on seven A549 cells from different cultured coverslips. Each cell was probed randomly with both tips at three points of the central region of the cell surface. Force curves were recorded with a maximum indentation of $\sim 1 \mu \mathrm{m}$. Five low-amplitude oscillatory cycles were also recorded in one of the three regions of the cell at an operating indentation of $\sim 500 \mathrm{~nm}$. The experimental protocol lasted $\sim 45 \mathrm{~min}$. Contact mode images were acquired after mechanical measurements in three cells with the pyramidal tip at $1 \mathrm{~Hz}$ and force set point $<0.5 \mathrm{nN}$.

\section{F. Data processing}

Each experimental $d-z$ curve obtained with the pyramidal tip was fitted with the blunted pyramid model [Eqs. (8) and (9)] expressed in terms of the displacement of the piezo $(z)$ and the deflection of the cantilever $(d)$, using

$$
F=k\left(d-d_{o f f}\right)
$$

and

$$
\delta=z-z_{c}-\left(d-d_{o f f}\right)
$$

where $k$ is the spring constant of the cantilever, $d_{\text {off }}$ is the deflection offset, and $z_{c}$ is the point of contact. Substituting Eqs. (11) and (12) into Eqs. (8) and (9), we obtained

$$
\begin{aligned}
d= & d_{o f f}+\frac{2 E}{k\left(1-\nu^{2}\right)}\left\{\left[z-z_{c}-\left(d-d_{o f f}\right)\right] a-\frac{2^{1 / 2} a^{2}}{\pi \tan \theta}\right. \\
& \times\left(\frac{\pi}{2}-\arcsin \frac{b}{a}\right)-\frac{a^{3}}{3 R_{c}}+\left(a^{2}-b^{2}\right)^{1 / 2} \\
& \left.\times\left(\frac{2^{1 / 2}}{\pi} \frac{b}{\tan \theta}+\frac{a^{2}-b^{2}}{3 R_{c}}\right)\right\}, \\
z-z_{c} & -\left(d-d_{o f f}\right)-\frac{a}{\tan \theta} \frac{2^{3 / 2}}{\pi}\left[\frac{\pi}{2}-\arcsin \left(\frac{b}{a}\right)\right]+\frac{a}{R_{c}} \\
& \times\left[\left(a^{2}-b^{2}\right)^{1 / 2}-a\right]=0 .
\end{aligned}
$$

Since there is no analytical solution for Eq. (14), the radius of contact $a$ was computed numerically using an interpolation routine.

The pyramidal tip data were also fitted with the ideal pyramidal tip model [Eq. (10)] expressed in terms of $z$ and $d$

$$
d=d_{o f f}+\frac{1}{2^{1 / 2}} \frac{E \tan \theta}{\left(1-\nu^{2}\right) k}\left[z-z_{c}-\left(d-d_{o f f}\right)\right]^{2} .
$$

The experimental $d-z$ curves obtained with the spherical tip were fitted with the spherical Hertz model [Eq. (5)] expressed in terms of $z$ and $d$

$$
d=d_{o f f}+\frac{4 E}{3\left(1-\nu^{2}\right) k} R^{1 / 2}\left[z-z_{c}-\left(d-d_{o f f}\right)\right]^{3 / 2},
$$

where $R$ is the tip radius. A nonlinear least-squares fit (Matlab 6.5, The MathWorks, Inc., Natick, MA, USA) was used to estimate $E, z_{c}$, and $d_{\text {off }}$ from each loading $d$ - $z$ curve. The fit included a clear noncontact region. For cell measurements, the first $\mu \mathrm{m}$ of the noncontact region was discarded. The average $E$ for each sample was computed using the last three curves at the three measurement points. The mean and standard deviation (SD) of these values were computed. To assess the dependence of $E$ on indentation, contact model equations [Eqs. (13), (15), and (16)] were solved for $E$. $E$ was computed from each force-indentation measurement point, using the values of $z_{c}$ and $d_{\text {off }}$ fitted with the $d-z$ curve. $E$ was computed at equally spaced indentation intervals by interpolation to allow averaging.

For low-amplitude oscillations around an operating indentation $\left(\delta_{0}\right)$, Eq. (8) can be approximated taking the first two terms of the Taylor expansion [20,21]. Using $G=E /[2(1$ $+v)$ ] and transforming to the frequency domain, $G^{*}(\omega)$ for a blunted pyramidal tip is

$$
G^{*}(\omega)=\frac{1-\nu}{4 a_{0}} \frac{F(\omega)}{\delta(\omega)},
$$

where $F(\omega)$ and $\delta(\omega)$ are the Fourier transforms of force and indentation, respectively, $\omega$ is the angular frequency $(\omega$ $=2 \pi f)$, and $a_{0}$ is the contact radius at $\delta_{0}$.

The same procedure was applied to obtain $G^{*}(\omega)$ for the spherical model [Eq. (5)] 


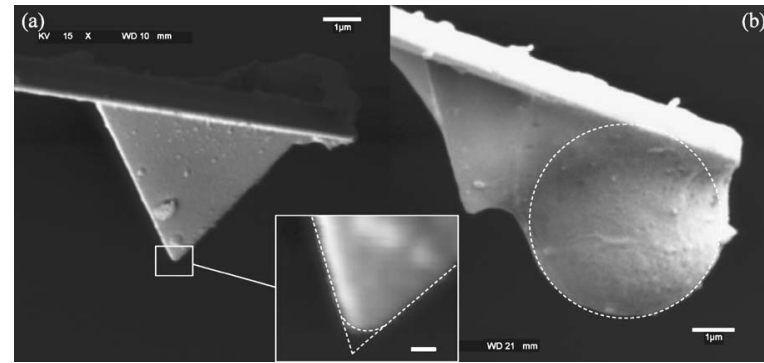

FIG. 2. Scanning electron micrographs of (a) pyramidal and (b) spherical tips used in agarose gels $($ bar $=1 \mu \mathrm{m})$. The inset shows the blunted pyramidal approximation $($ bar $=100 \mathrm{~nm})$.

$$
G^{*}(\omega)=\frac{1-\nu}{4\left(R \delta_{0}\right)^{1 / 2}} \frac{F(\omega)}{\delta(\omega)} .
$$

$G^{*}(\omega)$ was separated into its real (in-phase) and imaginary (out-of-phase) parts $\left[G^{*}(\omega)=G^{\prime}(\omega)+i G^{\prime \prime}(\omega)\right]$, defined as the storage and loss moduli, respectively. The viscoelastic behavior was characterized as the loss tangent $\left(G^{\prime \prime} / G^{\prime}\right)$. Before computing the FFT, recordings were digitally filtered with a high-pass filter (Butterworth, $0.1 \mathrm{~Hz}, 5$ poles), and multiplied by a Hanning window. Given the low oscillatory frequency applied $(0.3 \mathrm{~Hz})$, we neglected the hydrodynamic drag artifact [30].

\section{G. Statistics}

Data are reported as mean $\pm \mathrm{SD}$ unless otherwise stated. Differences in $E$ and $G^{*}$ values obtained using different tips were analyzed by Student's $t$-test. Statistical significance was assumed at $p<0.05$.

\section{RESULTS}

\section{A. Cantilever tips}

From the SEM images of the tips used in agarose (Fig. 2), we estimated a semi-included angle of $\theta=35.5^{\circ}$ for the pyramidal tip and a bead radius of $R=2.4 \mu \mathrm{m}$ for the spherical tip. We estimated $\theta=35.4^{\circ}$ and $R=2.5 \mu \mathrm{m}$ for the tips used on A549 cells. The pyramidal tips exhibited a blunted apex (Fig. 2). We modeled the actual tip geometry using the blunted pyramid model (tip defect $h=100 \mathrm{~nm}$ ) with the spherical cap merging tangential with the pyramid faces.

\section{B. Agarose gels}

The pyramidal and spherical tip cantilevers used on agarose gels had spring constants of $k=7.1 \pm 0.6 \mathrm{mN} / \mathrm{m}$ and $k$ $=7.9 \pm 0.6 \mathrm{mN} / \mathrm{m}$, respectively. Representative examples of $d-z$ curves taken on agarose are shown in Fig. 3. The loading and unloading curves were almost coincident. The fit of the model to the loading curve was excellent for both the pyramidal $\left[r^{2}=0.9994 \pm 0.0003\right.$, root-mean-square error (rms) $=4.46 \pm 3.06 \mathrm{~nm}]$ and spherical $\left(r^{2}=0.9991 \pm 0.0002, \mathrm{rms}\right.$ $=2.55 \pm 1.48 \mathrm{~nm}$ ) tips. The residual error plot (Fig. 3) showed only minor deviations from the models. Nevertheless, the models slightly underestimated the force in the vicinity of the contact point.
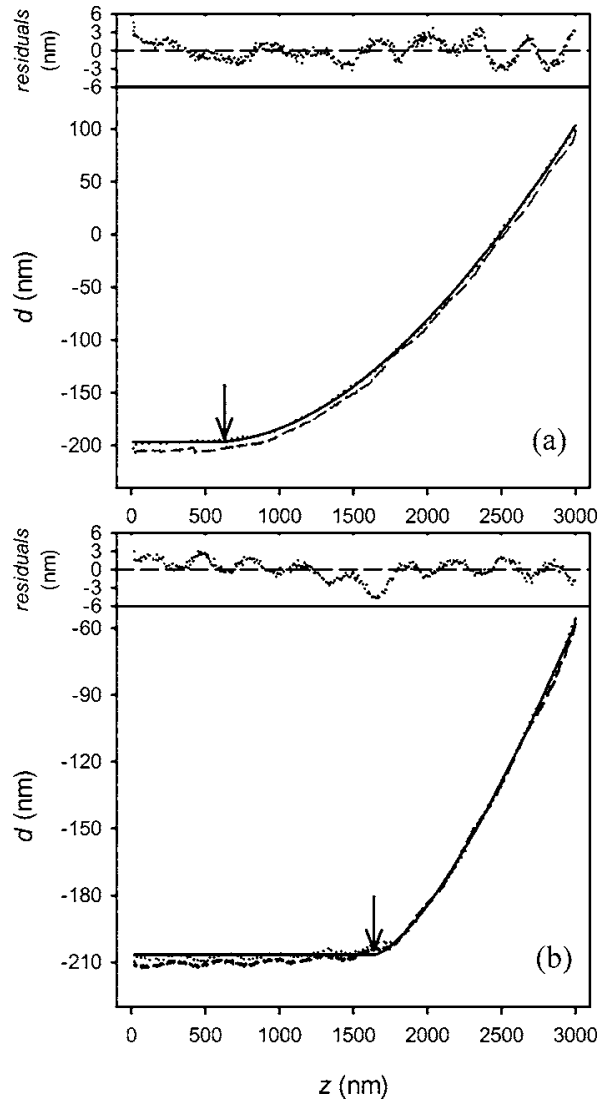

FIG. 3. Examples of deflection-distance $(d-z)$ curves measured on approach (dotted lines) and retraction (dashed lines) on agarose gels with (a) pyramidal and (b) spherical tips. Solid lines are the corresponding blunted pyramidal and spherical fits to approach curves. Fitted values were $E=0.76 \mathrm{kPa}, z_{c}=634 \mathrm{~nm}$, and $d_{\text {off }}$ $=-196 \mathrm{~nm}$, and $E=0.35 \mathrm{kPa}, z_{c}=1651 \mathrm{~nm}$, and $d_{o f f}=-207 \mathrm{~nm}$, respectively. Arrows indicate the contact point. Insets display residual errors.

The value of $E$ computed with the pyramidal tip $(1.38 \pm 1.36 \mathrm{kPa})$ was larger than that computed with the spherical one $(0.72 \pm 0.60 \mathrm{kPa}, p>0.05)$. We found a small coefficient of variation $(\mathrm{CoV})$ of $E$ obtained from consecutive curves acquired at the same point of the gel with the pyramidal $(3.9 \pm 2.4 \%)$ and spherical $(2.7 \pm 1.8 \%)$ tips. Larger variability for the pyramid $(10.9 \pm 10.5 \%)$ and the sphere $(13.6 \pm 4.9 \%)$ were found from measurements taken at different points of the same gel.

$E$ computed from the spherical tip exhibited a sharp fall at low indentations, reaching a plateau at $\sim 200 \mathrm{~nm}$ (Fig. 4). A similar indentation dependence was obtained with a plateau above $\sim 300 \mathrm{~nm}$ with the pyramidal tip using the blunted model [Eqs. (13) and (14)]. When employing the ideal pyramidal model [Eq. (15)], the plateau was shifted to slightly higher indentations $(\sim 400 \mathrm{~nm})$.

$G^{*}(\omega)$ was measured at comparable operating indentations of $563 \pm 92 \mathrm{~nm}$ and $475 \pm 134 \mathrm{~nm}$ for the pyramidal and spherical tips, respectively. The corresponding radii of contact were considerably smaller with the pyramid $(0.33 \pm 0.05 \mu \mathrm{m})$ than with the sphere $(1.06 \pm 0.15 \mu \mathrm{m}) . G^{\prime}$ was significantly higher when using the pyramidal tip $(0.43 \pm 0.20 \mathrm{kPa})$ than the spherical one $(0.17 \pm 0.05 \mathrm{kPa}, p$ 


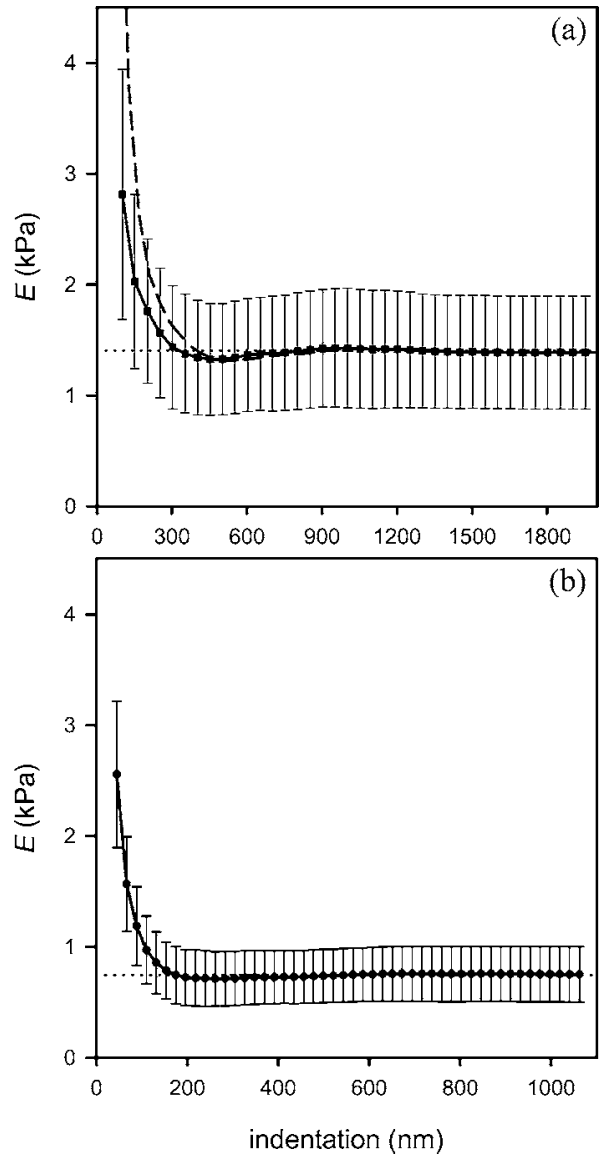

FIG. 4. Indentation dependence (mean \pm SE) of Young's modulus of agarose gels $(n=7)$ obtained with the (a) pyramidal and (b) spherical tips. Dashed line in (a) displays data fitted with the ideal pyramid model. Dotted lines are mean $E$ values obtained by fitting the whole $d-z$ curves.

$<0.05)$. In contrast, we found negligible values of $G^{\prime \prime}$ for both tips $\quad(0.02 \pm 0.05 \mathrm{kPa}$, pyramid; $-0.01 \pm 0.02 \mathrm{kPa}$, sphere).

\section{Alveolar epithelial cells}

The cantilevers used on A549 cells had spring constants of $k=6.3 \pm 0.4 \mathrm{mN} / \mathrm{m}$ and $k=7.9 \pm 0.6 \mathrm{mN} / \mathrm{m}$ for the pyramidal and spherical tips, respectively. The thickness of the cells in the probed regions was 5-6 $\mu \mathrm{m}$, and the $d-z$ curves of the cells exhibited considerable hysteresis (Fig. 5). We frequently observed deflection values below $d_{\text {off }}$ in the unloading curves, indicating cell-tip adhesion. The contact models fitted loading $d-z$ curves very well for the pyramidal [Eqs. (13) and (14), and $r^{2}=0.994 \pm 0.005$, rms $\left.=2.91 \pm 1.92 \mathrm{~nm}\right]$ and spherical [Eq. (16), $r^{2}=0.998 \pm 0.001, \quad \mathrm{rms}$ $=1.63 \pm 0.54 \mathrm{~nm}]$ tips. We obtained higher values of $E$ with pyramidal $(0.91 \pm 0.47 \mathrm{kPa})$ than with the spherical tip data $(0.47 \pm 0.18 \mathrm{kPa}, p<0.05)$. Repeated measurements obtained at the same point of the cell led to similar $\mathrm{CoV}$ for the pyramid $(5.3 \pm 3.8 \%)$ and the sphere $(3.1 \pm 2.8 \%)$. In contrast, the variability computed from different regions within the same cell rose to $41.9 \pm 21.5 \%$ (pyramid) and to $25.9 \pm 22.7 \%$ (sphere). Young's modulus of cells showed an indentation
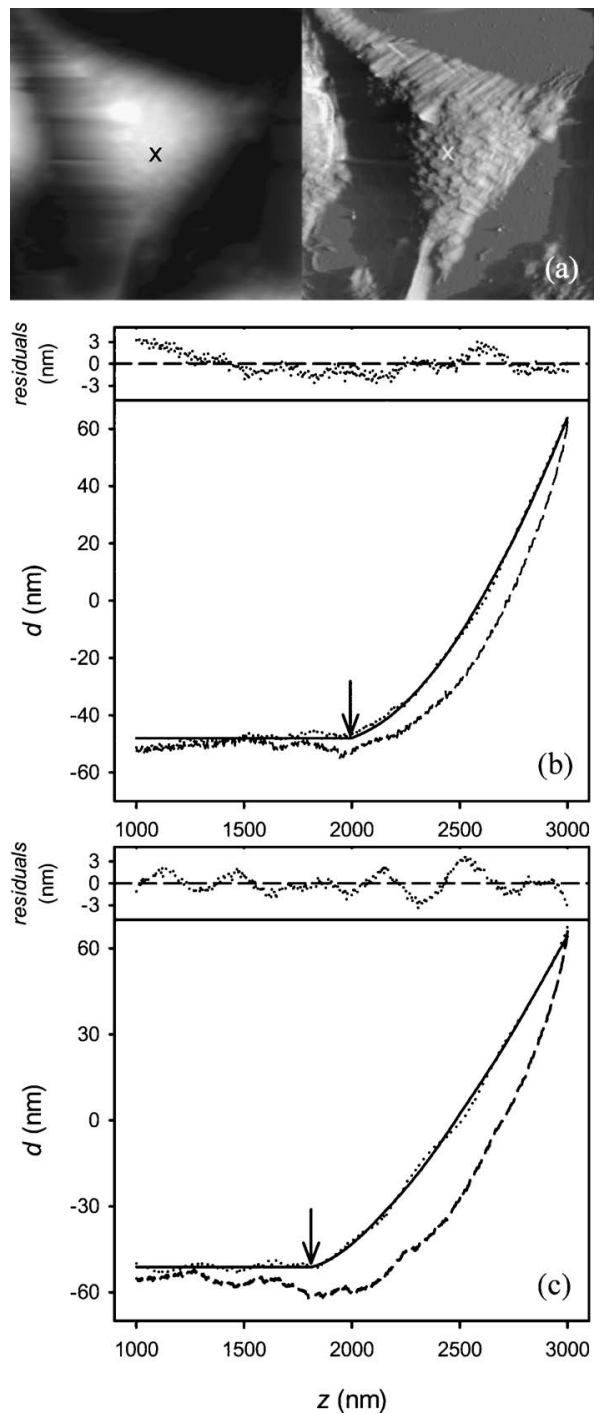

FIG. 5. (a) Height (left) and deflection (right) images of an A549 cell obtained with the pyramidal tip in contact mode (scan size $=50 \mu \mathrm{m}$ ). Gray level ranges $6 \mu \mathrm{m}$ (left) and $50 \mathrm{~nm}$ (right). (b) and (c) Examples of deflection-distance $(d-z)$ curves measured on approach (dotted lines) and retraction (dashed lines) on the cell with (b) pyramidal and (c) spherical tips. Fitted values were $E$ $=1.31 \mathrm{kPa}, z_{c}=1997 \mathrm{~nm}$, and $d_{o f f}=-49 \mathrm{~nm}$, and $E=0.36 \mathrm{kPa}, z_{c}$ $=1812 \mathrm{~nm}$, and $d_{o f f}=-51 \mathrm{~nm}$, respectively. Arrows indicate the contact point. Insets display residual errors. The crosses in (a) indicate the points where the pyramidal force curve was obtained.

dependence similar to that of gels (Fig. 6), reaching the plateau at comparable indentation depths.

$G^{*}(\omega)$ was measured at operating indentations of $716 \pm 255 \mathrm{~nm}(a=0.41 \pm 0.13 \mu \mathrm{m})$ with the pyramid and $541 \pm 225 \mathrm{~nm}(a=1.13 \pm 0.29 \mu \mathrm{m})$ with the sphere. With the pyramidal tip, we found $G^{\prime}=0.32 \pm 0.16 \mathrm{kPa}$ and $G^{\prime \prime}$ $=0.07 \pm 0.03 \mathrm{kPa}$, corresponding to a loss tangent of $0.23 \pm 0.12$. Lower values of $G^{\prime}=0.15 \pm 0.07 \mathrm{kPa}(p<0.05)$ and $G^{\prime \prime}=0.03 \pm 0.02 \mathrm{kPa}(p<0.05)$ were obtained with the spherical tip, although no significant difference was found in the loss tangent $(0.21 \pm 0.07)$. 

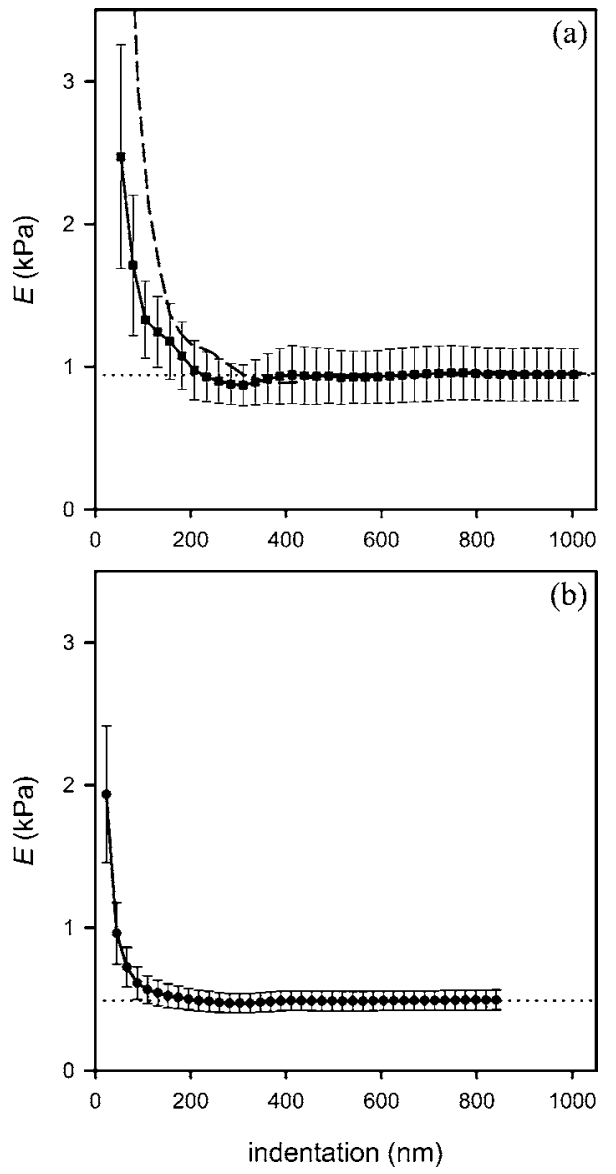

FIG. 6. Indentation dependence (mean \pm SE) of Young's modulus of alveolar epithelial cells $(n=7)$ obtained with the (a) pyramidal and (b) spherical tips. Dashed line in (a) displays data fitted with the ideal pyramid model. Dotted lines are mean $E$ values obtained by fitting the whole $d-z$ curves.

\section{DISCUSSION}

We have developed a contact model to describe the forceindentation relationship of a blunted pyramid indenting an elastic half-space. The model was extended for application to viscoelastic samples subjected to small-amplitude oscillations. Soft agarose gels probed with AFM blunted pyramidal tips showed a fall in $E$ at low indentations, reaching a constant value for depths larger than $\sim 300 \mathrm{~nm}$. A similar indentation dependence of $E$ was observed when the gels were probed with the spherical tip. Nevertheless, $E$ and $G^{\prime}$ measured at deep indentations with the sphere were twofold lower than those found with the pyramid. The gels exhibited an elastic behavior with almost coincident loading and unloading force curves and negligible values of $G^{\prime \prime}$. The dependence of $E$ on indentation in cells paralleled that found in the gels. Cells exhibited viscoelastic behavior with a $G^{\prime \prime} / G^{\prime}$ ratio $\sim 1 / 4$.

The mathematical approach used to develop the blunted pyramidal model was based on Betti's reciprocal theorem and the Rayleigh-Ritz approximation [26]. The Betti reciprocal theorem allowed us to relate the total force applied by the pyramidal tip to the pressure distribution of a flat tip with the same contact area [Eq. (1)]. Since this equation cannot be

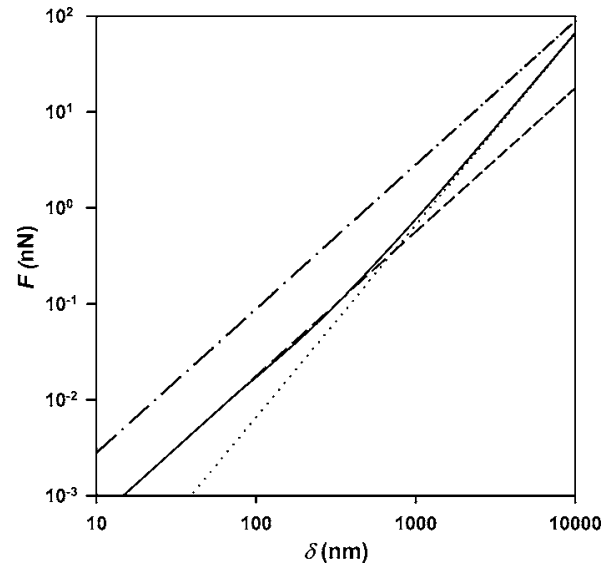

FIG. 7. Force-indentation $(F-\delta)$ relationship for blunted pyramidal, ideal pyramidal, and spherical models on an elastic half-space with Young's modulus $E=1 \mathrm{kPa}$. Solid line is the blunted pyramidal model with semi-included angle $\theta=35^{\circ}$ and a spherical cap of radius $R_{c}=100 \mathrm{~nm}$ merging tangential with the faces. Dotted line is the ideal pyramidal model with $\theta=35^{\circ}$ (slope $=2$ ). Dashed line is the spherical model of radius $R=100 \mathrm{~nm}$ (slope $=3 / 2$ ). Dasheddotted line is the spherical model with $R=2500 \mathrm{~nm}$ (slope=3/2). The blunted pyramidal model matches the spherical model of $R$ $=100 \mathrm{~nm}\left(R_{c}=R\right)$ for indentations smaller than $80 \mathrm{~nm}\left(\delta<2 h^{*}\right)$ and approaches the ideal pyramidal model for deeper indentations. The difference is $<15 \%$ for indentations deeper than $1000 \mathrm{~nm}$.

solved analytically, we made use of the Rayleigh-Ritz approach in determining the best elliptical approximation to the actual contact area. This approximation is useful when the main interest is to compute $F(\delta)$ and not the pressure distribution, which requires a more complex mathematical development. Barber and Billings used this procedure to obtain the $F(\delta)$ of an ideal three-sided pyramidal tip [27]. Their solution differed by only $13 \%$ when compared with numerical computations. Given that the intervals of integration decrease with the number of sides, a lower error for a foursided pyramid was estimated. In fact, the solution we found for an ideal four-sided pyramid [Eq. (10)] agrees within 6\% with the equation derived by Bilodeau based on the stress distribution of a cone modified by the singularities at the lateral edges of the pyramid [19]. By imposing a smooth transition from the spherical cap to the pyramid faces, the faces must be slightly curved near the cap. This curvature leads to a smaller cross-sectional area compared with that of flat faces (Fig. 1). At an indentation of $\sim 300 \mathrm{~nm}(\theta$ $\left.=35^{\circ}, R_{c}=100 \mathrm{~nm}\right)$, the difference between the flat and curved surfaces is $\sim 8 \%$ and tends to zero at deeper indentations. Assuming that the force is proportional to the area, we estimate a similar difference in $E$.

The blunted pyramidal model matches the spherical model with a radius equal to that of the spherical cap $(R$ $=R_{c}$ ) for indentations where the sample only contacts the spherical cap $\left(\delta<2 h^{*}\right)$, and at deeper indentations, the blunted pyramidal model approaches the ideal pyramidal model smoothly (Fig. 7). Note that the $n$-sided blunted pyramidal model [Eqs. (6) and (7)] is easily solvable for the variety of pyramidal geometries commercially available. The semi-included angle measured by SEM was in very good 
agreement with its nominal value $\left(35^{\circ}\right)$. This was expected from the stability of the fabrication process [31] and suggests that the nominal $\theta$ could be used to compute the blunted pyramidal model. However, the radius of the spherical cap measured by SEM $\left(R_{c}=123 \mathrm{~nm}\right)$ was twofold larger than its nominal value $(\sim 50 \mathrm{~nm})$. Using the nominal radius would overestimate $E$ by $\sim 65 \%$ for low indentations $\left(\delta<2 h^{*}\right)$. The difference would tend to zero for deeper indentations, being $<1 \%$ for $\delta>1 \mu \mathrm{m}$. Thus, accurate measurements at low indentations with a blunted pyramidal tip would require precise characterization of tip bluntness. It should also be pointed out that the solution for an $n$-sided blunted pyramid [Eqs. (6) and (7)] tends to that of a blunted cone when $n$ $\rightarrow \infty$ [17]. When, in addition, $b \rightarrow 0$, the solution corresponds to that of an ideal cone [32].

AFM systems tilt the cantilever by $10^{\circ}-20^{\circ}$ so that the beams of the cantilever do not touch the sample. Heim and co-workers studied the contribution of the inclination angle $(\beta)$ to the deflection of the cantilever and suggested a correction factor [33]. Given the cantilever and tip dimensions that we used (320 $\mu \mathrm{m}$ cantilever length, 4-5 $\mu \mathrm{m}$ tip height and $12^{\circ}$ tilt), Heim's correction factors were $<0.5 \%$ for both the spherical and pyramidal tips and were thus neglected. Moreover, current contact models assume normal indentation of the tip on the sample surface. Costa and Yin [34] discussed the effect of tip inclination on $F(\delta)$ for an ideal cone. They suggested that the $F(\delta)$ of a tilted conical tip lies between the responses for semi-included angles $\theta+\beta$ and $\theta$ $-\beta$. The force of a regular pyramid in normal indentation is the addition of the force applied to each face. We assume that the total force of a tilted pyramid is composed of the contributions of each face, weighted by the areas of their cross sections. In addition, we consider that each face of the tilted pyramid follows the $F(\delta)$ of an ideal pyramid [Eq. (10)] with semi-included angle $\theta \pm \beta$ (faces parallel to the inclination axis) or $\theta$ (faces perpendicular to the inclination axis). From this approach, we estimate an apparent increase in $E$ of $\sim 10 \%$ when tilting an ideal four-sided pyramid $\left(\theta=35^{\circ}\right)$ by $12^{\circ}$. Assuming a similar behavior for the blunted pyramidal model, our measurements taken with the tilted pyramidal tip overestimated $E$ by $\sim 10 \%$.

Reliable estimation of mechanical parameters from $d-z$ curves requires accurate determination of the contact point $[21,35,36]$. In general, the contact point is visually defined at the point where cantilever deflection starts to rise. However, visual determination of the contact point is especially difficult in soft samples that exhibit a smooth increase in cantilever deflection at low indentations (Figs. 3 and 5). To define the contact point with an objective procedure, we simultaneously estimated $E$ and the location of the contact point $\left(z_{c}, d_{o f f}\right)$ by fitting $d-z$ curves that included a noncontact region. The fitting was carried out in the approaching curve to avoid the adhesion events observed in the retraction curves, especially in cells (Fig. 5). The quality of the fit with the two tips was excellent in gels and cells. It should be noted, however, that both models slightly underestimated cantilever deflection in the vicinity of the contact point (Figs. 3 and 5).

We first assessed the suitability of AFM pyramidal tips for probing mechanics in soft agarose gels. We used agarose gels as a reference sample since different techniques have shown that their mechanical properties are elastic, linear, homogeneous, and isotropic [25,37]. Moreover, we made the gel samples $\sim 500 \mu \mathrm{m}$ thick to avoid the effect of the hard substrate. These features are required to fulfill the assumptions used to derive tip contact models. The high repeatability of $E$ computed from consecutive curves recorded at the same point $(\mathrm{CoV}<4 \%)$ indicates stability of the sample and absence of permanent deformation. The homogeneity of the gel was reflected in the low variation of $E$ among different points of the sample $(\mathrm{CoV}<10 \%)$. The near coincidence of the approach and retraction $d-z$ curves confirmed the elastic behavior of our gel samples. Measurement of the complex shear modulus allows us to assess the frequency-domain expansion of the blunted model [Eq. (17)]. In agreement with the elastic behavior, we found negligible values of $G^{\prime \prime}$. Moreover, the $E / G^{\prime}$ ratio obtained for the pyramidal tip (3.21) was similar to that of a pure elastic material $\left(E / G^{\prime}=3, v\right.$ $=0.5$ ). The consistency between $G^{*}$ and $E$ lends support to the validity of the complex expansion of the blunted pyramidal model [Eq. (17)]. Taking into account the discrepancies between microrheological and macrorheological measurements [38], our data of $G^{\prime}$ compare well with the value of $0.52 \mathrm{kPa}$ derived by Normand and co-workers [37] from conventional macroscopic rheology measurements on the same type of agarose at $0.3 \%$. The value of $E$ obtained by fitting the whole $d-z$ curves recorded with the pyramidal tip was twofold higher than that computed with the spherical tip. This difference could reflect uncertainties of the calibration procedure of the cantilevers, which assumes a simple harmonic oscillator model for cantilever mechanics. Nevertheless, $E$ and $G^{*}$ obtained with the two tips are in reasonable agreement, bearing in mind that the difference is comparable to the variability of the gel samples.

To test the range of validity of the blunted pyramidal model, we computed the indentation dependence of $E$ in agarose gels (Fig. 4). For a linear material, $E$ should be constant over the whole indentation range. Except at low indentations $(<300 \mathrm{~nm}), E$ computed with the blunted pyramidal tip showed a plateau up to the deepest indentation reached $(\sim 2 \mu \mathrm{m})$. It is noteworthy that fitting the ideal pyramid model also results in the same plateau, but slightly shifted to deeper indentations. Therefore, for deep indentations, $E$ can be more easily estimated by using Eq. (10). It has been suggested that the linear assumption of the Hertz theory fails for the large strains produced by pyramidal tips [22]. To minimize strain, probing the sample with a microsphere has been proposed [21-23]. The plateau obtained when probing gels and polymers with a microsphere attached to the cantilever has been taken as evidence of the validity of the spherical contact model [21]. We found that the pyramidal tip exhibited a constant regime similar to that observed with the spherical tip. The linear regime displayed by the pyramidal tip is in agreement with finite element analysis, indicating that high strains applied by sharp tips have only second-order effects on the indentation response for linear materials [34]. Moreover, it should be noted that the blunted profile of conventional AFM pyramidal tips reduces the strain in the vicinity of the apex.

Agarose gels exhibited a parallel fall in $E$ at low indentations with both tips. This behavior has been reported in gels 
and living cells. Large and scattered values of $E$ at low indentations have been attributed to experimental noise, to nonlinear elastic behavior of the samples [18], and to uncertainties in the contact point determination [21-23]. However, we averaged data from several measurements (Fig. 4) to cancel out the effect of random experimental noise. Moreover, the plateau of $E$ obtained in a wide range of indentations with both tips indicates intrinsic linear mechanical properties of the gel. $E$ estimated at low indentations is very sensitive to uncertainties in the contact point. In fact, the large $E$ we found at low indentations could be explained by an error of $1-2 \mathrm{~nm}$ in $d_{o f f}$. To avoid ambiguities associated with visual determination, we computed the contact point by a robust fitting procedure. The slight but systematic underestimation of cantilever deflection by the two contact models in the vicinity of the contact point (Fig. 3) suggests that $E$ at low indentations reflects features of tip-surface interaction. This underestimation of cantilever deflection can be interpreted as a repulsive force. Assuming that agarose and the tip are electrically neutral, a repulsive force could arise from steric interactions of free-end agarose chains forming brushlike arrangements in the gel-liquid interface [39]. The similar fall of $E$ at low indentations and the deeper linear regime exhibited by the pyramidal and spherical tips on the agarose gels suggest that both cantilevers are suitable for probing the mechanical properties of uniform and thick soft samples.

The performance of the tips in cells was in agreement with that observed in gels. Both tips displayed large values of $E$ at low indentations, followed by a sharp fall reaching a plateau at $\sim 200 \mathrm{~nm}$ that extended to the deepest indentation obtained. Similar indentation dependence has been reported in other cell types $[18,21,23]$. In accordance with the results found in gels, the plateau value of $E$ estimated with the two tips differed by a factor of $\sim 2$. The constant $E$ observed over a broad indentation range indicates a fairly linear behavior of cell mechanics. However, the sharp rise of $E$ at low indentions deviated from the linear behavior. This surface singularity may reflect the effect of steric forces associated with the brushlike glycocalix of living cells $[40,41]$. The repeatability of consecutive measurements at the same point taken with both tips $(\sim 5 \%)$ was as good as in the gels. On the other hand, the larger variability among cell regions reflected a more heterogeneous mechanical behavior of the cells. Moreover, the higher heterogeneity observed with the pyramidal tip reflects improved lateral resolution. In contrast to the near-elastic behavior of agarose gels, the cells exhibited viscoelastic properties, with the oscillatory response dominated by elastic stresses. Similar viscoelastic behavior has also been observed in the same cell type twisting magnetic beads attached to the cell surface [42]. Despite the higher values of the storage and loss moduli measured with the pyramidal tip, their ratio $\left(G^{\prime \prime} / G^{\prime}=0.23 \pm 0.12\right)$ was almost coincident with that obtained with the spherical tip $(0.21 \pm 0.07)$. This indicates that both tips differed only in a scale factor. The agreement between the $G^{\prime \prime} / G^{\prime}$ ratio obtained with both tips lends further support to the complex expansion of the blunted pyramidal model [Eq. (17)].

The use of a pyramidal tip allows us to obtain highresolution images of the cell and to measure cell mechanics with the same probe. The resolution of the images enables us to observe cell topography in detail and to identify cytoskeleton fibers and other submembrane structures (Fig. 5). A recent work compared the image resolution obtained with pyramidal and spherical tips on porcine articular cartilage [25]. Individual collagen fibers observed with pyramidal tips were not resolved with microspheres, thus reflecting the poor image resolution of such tips. The linear regime of cell mechanics was clearly reached with the pyramidal tip for indentations of $\sim 300 \mathrm{~nm}$. The effective contact radius of the pyramidal tip at this indentation was $\sim 200 \mathrm{~nm}$, which provides an estimation of the lateral resolution of the mechanical measurements. The use of a single cantilever for imaging and mechanics is an important advantage, which provides precise correlation between viscoelastic mapping and cell structure. We probed the cells at points of 5-6 $\mu \mathrm{m}$ thickness, reaching indentations up to $\sim 1 \mu \mathrm{m}$. We did not observe hardening effects due to the underlying hard substrate, indicating that the contact model can be reliably applied for indentations up to $\sim 20 \%$ of cell thickness. Consequently, the pyramidal tip can probe cell mechanics with indentations up to $300 \mathrm{~nm}$ for cell thickness $>1.5 \mu \mathrm{m}$. This condition is maintained over a large region of the cell body of the lung epithelial cells. However, measurements at the thin cell periphery would require correction for the influence of the hard substrate $[22,23]$.

In conclusion, we developed a contact model of a blunted pyramidal tip to compute viscoelastic properties of soft samples indented with conventional AFM pyramidal tips. Agarose gels and living cells probed with a blunted pyramidal tip exhibited elastic behavior with large values of Young's modulus for indentations lower than $\sim 300 \mathrm{~nm}$ and a linear regime at deeper indentations. This indentation dependence paralleled that obtained with spherical tips, indicating that the larger strains applied by the pyramidal tip at the apex have minor effects on the force-indentation response. However, Young's modulus computed in the linear regime with the two tips differs by a factor of $\sim 2$. Cells exhibited viscoelastic behavior, with almost coincident values of the loss tangent obtained with both tips. Cells displayed a linear regime for indentations up to $\sim 20 \%$ of cell thickness. This allows probing viscoelasticity with the pyramidal tip in a broad central region of the cell body with negligible influence of the hard substrate. Our results suggest that pyramidal tips commonly used for AFM imaging are suitable for probing mechanical properties of soft polymer gels and living cells. The use of a single cantilever enables us to precisely correlate imaging and viscoelastic mapping of living cells.

\section{ACKNOWLEDGMENTS}

The authors wish to thank Miguel Rodríguez for his technical assistance. This work was supported in part by grants from the Ministerio de Ciencia y Tecnologia (Grant Nos. SAF 2002-03616 and SAF 2003-01334) and the Ministerio de Sanidad y Consumo (Grant Nos. FIS-PI040929, Red GIRA-G03/063 and Red RESPIRA-C03/11). 
[1] C. Rotsch and M. Radmacher, Biophys. J. 78, 520 (2000).

[2] M. Heuberger, G. Dietler, and L. Schlapbach, J. Vac. Sci. Technol. B 14, 1250 (1996).

[3] H. X. You and L. Yu, Methods in Cell Science 21, 1 (1999).

[4] M. Tortonese, IEEE Eng. Med. Biol. Mag. 16, 28 (1997).

[5] S. R. Heidemann and D. Wirtz, Trends Cell Biol. 14, 160 (2004).

[6] E. Henderson, P. G. Haydon, and D. S. Sakaguchi, Science 257, 1944 (1992).

[7] G. T. Charras and M. A. Horton, Biophys. J. 82, 2970 (2002).

[8] R. Lal et al., Am. J. Physiol.: Cell Physiol. 269, C275 (1995).

[9] H. X. You, J. M. Lau, S. Zhang, and L. Yu, Ultramicroscopy 82, 297 (2000).

[10] H. Huang, R. D. Kamm, and R. T. Lee, Am. J. Physiol.: Cell Physiol. 287, C1 (2004).

[11] H. Hertz, J. Reine Angew. Math. 92, 156 (1881); Miscellaneous Papers (Macmillan, London, 1896), p. 146.

[12] K. L. Johnson, Contact Mechanics, 1st ed. (Cambridge University Press, Cambridge, U.K., 1985).

[13] M. Radmacher, M. Fritz, and P. K. Hansma, Biophys. J. 69, 264 (1995).

[14] M. Radmacher, R. W. Tilmann, and H. E. Gaub, Biophys. J. 64, 735 (1993).

[15] S. G. Shroff, D. R. Saner, and R. Lal, Am. J. Physiol.: Cell Physiol. 38, C286 (1995).

[16] H. W. Wu, T. Kuhn, and V. T. Moy, Scanning 20, 389 (1998).

[17] B. J. Briscoe, K. S. Sebastian, and M. J. Adams, J. Phys. D 27, 1156 (1994).

[18] A. B. Mathur et al., J. Biomech. 34, 1545 (2001).

[19] G. Bilodeau, Trans. ASME, J. Appl. Mech. 59, 519 (1992).

[20] J. Alcaraz et al., Biophys. J. 84, 2071 (2003).

[21] R. E. Mahaffy, C. K. Shih, F. C. MacKintosh, and J. Kas, Phys. Rev. Lett. 85, 880 (2000).
[22] E. K. Dimitriadis et al., Biophys. J. 82, 2798 (2002).

[23] R. E. Mahaffy et al., Biophys. J. 86, 1777 (2004).

[24] B. Shoelson et al., Biophys. J. 87, 2768 (2004).

[25] M. Stolz et al., Biophys. J. 86, 3269 (2004).

[26] A. C. Ugural and S. K. Fenster, Advanced Strength and Applied Elasticity, 2nd ed. (Elsevier Science, New York, 1987).

[27] J. R. Barber and D. A. Billings, Int. J. Mech. Sci. 32, 991 (1990).

[28] J. L. Hutter and J. Bechhoefer, Rev. Sci. Instrum. 64, 1868 (1993).

[29] R. W. Stark, T. Drobek, and W. M. Heckl, Ultramicroscopy 86, 207 (2001).

[30] J. Alcaraz et al., Langmuir 18, 716 (2002).

[31] T. R. Albrecht, S. Akamine, T. E. Carver, and C. F. Quate, J. Vac. Sci. Technol. A 8, 3386 (1990).

[32] I. N. Sneddon, Int. J. Eng. Sci. 3, 47 (1965).

[33] L. O. Heim, M. Kappl, and H. J. Butt, Langmuir 20, 2760 (2004).

[34] K. D. Costa and F. C. P. Yin, J. Biomech. Eng. 121, 462 (1999).

[35] E. A-Hassan et al., Biophys. J. 74, 1564 (1998).

[36] A. Touhami, B. Nysten, and Y. F. Dufrêne, Langmuir 19, 4539 (2003).

[37] V. Normand et al., Biomacromolecules 1, 730 (2000).

[38] F. G. Schmidt, B. Hinner, and E. Sackmann, Phys. Rev. E 61, 5646 (2000).

[39] H. J. Butt et al., Langmuir 15, 2559 (1999).

[40] M. Fritz, M. Radmacher, and H. E. Gaub, Biophys. J. 66, 1328 (1994).

[41] I. Lee and R. E. Marchant, Colloids Surf., B 19, 357 (2000).

[42] X. Trepat et al., Am. J. Physiol-Lung Cell Mol. Physiol 287, L1025 (2004). 